\title{
MEPAG Steering Committee Diversity, Equity, Inclusion and Accessibility White Paper Statement
}

\author{
Prepared on behalf of the Mars Exploration Program Analysis Group \\ (MEPAG) \\ by the MEPAG Steering Committee as Input to the \\ 2023-2032 Planetary Science and Astrobiology Decadal Survey
}

Contact information: Dr. R. Aileen Yingst, MEPAG chair, Planetary Science Institute; 920-360-3627; yingst@psi.edu

14 September 2020

\begin{abstract}
Also posted on the MEPAG web site, and may be referenced as follows:
MEPAG (2020), Diversity, Equity, Inclusion and Accessibility White Paper Statement, R.A. Yingst, ed., 1 p. white paper posted Sept. 14, 2020 by the Mars Exploration Program Analysis Group (MEPAG) at http://mepag.jpl.nasa.gov/decadal/index.html.
\end{abstract}


Diversity, Equity, Inclusion and Accessibility (DEIA) are crucial factors in the strength, flexibility, and robustness of our profession. Equity, inclusion of, and accessibility for the broadest range of individuals, all work to foster the greatest level of diversity. Diversity itself ensures that new ideas, varied experiences, and unique perspectives are integrated fully into the discipline, leading to more creative solutions and approaches. DEIA efforts within the Planetary Science community have become a focal point of several NASA Assessment/Analysis Groups (AGs). The EDI working group was formed by the AGs, and its steering committee consists of members of each AG steering committee and several affinity groups. The working group has helped gather community input in the form of a series of professional white papers for the 20232032 Planetary Science Decadal Survey, including an overview paper by Richey and Milazzo, and nearly twenty additional papers. These white papers have been submitted by their individual authors in support of the Survey's Statement of Task Part 9: The state of the profession including issues of diversity, inclusion, equity, and accessibility, the creation of safe workspaces, and recommended policies and practices to improve the state of the profession. The white papers produced by this working group span a number of issues that have profound import and value to the community; each is important on its own, and together the body of white papers represents a detailed assessment of the state of the discipline in terms of how and how well it represents the broad diversity of our citizenry.

The Decadal Survey, through its final report, charts the course of the discipline for the next ten years; the many submitted white papers feed into that report, but to a large extent it is the resulting report that will be read and referenced. Thus, our fundamental concern is that information from these white papers be effectively assimilated into this document. In concert with MEPAG's intent that the Decadal Survey should hear from the community, we therefore endorse without prioritization the concept and prime intent of these DEIA white papers. We further encourage in the strongest terms careful attention to these papers and the issues they address, and request considered action on these issues by the Decadal Survey in their final report. The Decadal Survey is in the position to help our profession turn talk into the action that will ensure that the doors of our profession are wide open and welcoming for the betterment of the Planetary Science and Astrobiology fields. 